\title{
Rhizomatic Strategizing in Digital Transformation: A Clinical Field Study
}

\author{
Johan Magnusson \\ SCDI, University of \\ Gothenburg \\ johan.magnusson@ \\ gu.se
}

\author{
Andreas Nilsson \\ SCDI, University of \\ Gothenburg \\ andreas.nilsson@ait. \\ gu.se
}

\author{
Tomas Lindroth \\ SCDI, University of \\ Gothenburg \\ tomas.lindroth@ait. \\ gu.se
}

\author{
Jwan Khisro \\ SCDI, University \\ of Gothenburg \\ jwan.khisro@ait.g \\ $\underline{\text { u.se }}$
}

\author{
Kristian Norling \\ SCDI, University of \\ Gothenburg \\ kristian.norling@ait. \\ gu.se
}

\begin{abstract}
Most organizations today are involved in transformation initiatives; this has led to a burgeoning interest in the phenomenon of digital transformation strategy. Here, we present the findings of a clinical field study of a large Swedish municipality that has been involved in an ambitious digital transformation program since 2017. Despite explicitly not having a formal strategy, the organization utilizes a pseudoformalized and emergent strategy-as-practice for digital transformation that involves a set of key traits that have emerged over the years. We show how these traits have emerged and theorize on how the process can be understood as rhizomatic strategizing. The strategy emerges over time through a series of de- and reterritorializations, expanding through amalgamating new concepts into a strategy-as-practice for digital transformation.
\end{abstract}

\section{Introduction}

Organizations are experiencing significant pressure to increase their utilization of digital solutions to transform operations and ensure sustained relevance $[25 ; 31 ; 35]$. A multitude of studies have highlighted the central traits of this often grand-scale change, which is intermittently referred to as digital transformation and digitalization.

As organizations increasingly emphasize digital transformation, they deploy what Chanias et al. [6] referr to as digital transformation strategies, which offer the roadmap and directions for executing digital transformation. As noted by Vial [35], these strategies differ in terms of how digital solutions become essential to the transformation of the organization. While some strategies emphasize digitalizing internal operations, others are more directed toward external parties like customers or citizens. While some strategies look to exploit existing opportunities, others are more focused on exploring new value offerings and capturing opportunities [1].

In studying strategies, the strategy-as-practice movement $[20 ; 38]$ highlights the fact that strategy is a question of action, not plans. It is, in other words, a question of what an organization does rather than what their steering documents and/or strategic plans say and intend. This perspective on strategy infers that the study of strategy needs examine how an organization works, i.e., a turn to practice [5]. In the case of digital transformation strategy, the question becomes one of how an organization enacts its digital transformation [41], i.e., in essence, an emergent process.

This study approaches the digital transformation strategy-as-practice with the goal of answering the research call of Chanias et al. [6] regarding understanding the process of strategizing in pre-digital organizations. We study the creation and evolution of digital transformation strategy, assuming that digital transformation is a new phenomenon for the organization in question, by answering the following research question:

How does a digital transformation strategy-aspractice emerge over time?

This study contributes through an empirical account of the emergence of a digital transformation strategy in a large Swedish municipality and offers an interpretation of the process as an example of rhizomatic strategizing, i.e., borrowing ideas from changes in culture as depicted by Deleuze \& Guattari [9].

The remainder of the paper is organized as follows. First, we present precursory findings from digital transformation strategy-as-practice and rhizomatic strategizing. We then present the method of the study. A chronologically organized overview of the emergence of the digital transformation strategy in the municipality is then presented, followed by a discussion that theoretically examines the case as an instance of rhizomatic strategizing. 


\section{Precursory findings and theoretical framing}

\subsection{Digital transformation strategy-as-practice}

Digital transformation is a phenomenon that involves deep societal change $[14 ; 30 ; 35]$. It is also a continuous process of business improvements through the adoption and use of digital solutions [6;37] that facilitate, for example, streamlining operations, increased value creation, or increased customer satisfaction $[13 ; 27 ; 30]$.

According to Chanias et al. [6], a digital transformation strategy provides the organization with an answer regarding how a digital strategy may be developed and implemented across the entire organization. In other words, the digital transformation strategy is a necessary element for organizations that aspire to increase their utilization of digital technology $[44 ; 45 ; 46]$. In this regard, it is separated from previous notions, such as the digital business strategy as proposed by Bharadwaj et al. [48], because it counteracts the tendency to regard strategies related to IT and digital at the functional level, i.e., subject to alignment with the dominant business strategy. The digital is inherently integrated into both strategy and operations and must therefore be fused rather than aligned. As Bharadwaj et al. [48, p.472] stated, the digital busines strategy is, "...formulated and executed by leveraging digital resources to create differential value." This highlights the difference between the digital business strategy and the digital transformation strategy; the former is the strategy of leveraging digital resources (asset-focused) while the latter is the strategy of transforming the enterprise (intent-focused). We argue that organizations need both of these strategies as the digital becomes integral, but that the inert nature of organizations warrants the increased need for a digital transformation strategy and a conceptual disassociation from digital strategy [6, 47].

In the study of strategy, the strategy-as-practice movement challenges the underlying assumption of strategic management, which is that strategies are formulated and executed in an instrumental, mechanistic manner $[16 ; 17 ; 42]$. Rather, the core idea of the strategy-as-practice movement is that strategies are emergent and enacted in a process of constant (re-) configuration. In this vein, the strategy-as-practice movement affords little correspondence between the strategy as depicted in a strategic steering document and the actual strategy-in-action of an organization. Strategy is instead understood as day-to-day activities, which are referred to as strategy-as-practice or strategizing [39; $19 ; 40]$. It recognizes the activities of people, processes, and practices as bearers of strategy $[39 ; 21]$ through strategizing being, “... a socially accomplished, situated activity arising from the actions and interactions of multiple level actors" [20, p. 6]. In other words, strategy may and should be studied through activities rather than documents, through action rather than expressed intent.

In their study of the emergence of a digital transformation strategy in a non-digital financial service provider, Chanias et al. [6] showed that the digital transformation strategy is continuously emergent. Their study offered several key insights regarding the future study of digital transformation strategies, such as the strategy being customer/business-centric rather than technology-centric, its scope and implications being organization-wide, the emergent process being open and involving bottom-up influence, and the strategy never being entirely finalized. We utilize these findings as theoretical entry-points into understanding digital transformation strategy-as-practice.

\subsection{Rhizomatic strategizing}

"Where are you going? Where are you coming from? What are you heading for? These are totally useless questions." p.25

In the seminal work of Deleuze and Guattari [9], the rhizome, or mass of roots, was proposed as an alternative model for understanding the emergence of culture. As compared to arborescent models, which are hierarchal, causal, and chronological and come from a starting that branches into a tree-like structure, rhizomatic models capture an unhinged and un-directed emergent process of culture formation, highlighting the horizontal rather than the vertical.

Rhizomatic models therefore create a new perception of emergent processes in organizations. Gone is the teleologically-laden assumption of causal hierarchal chains and management agency; culture is closer to chaos than to order and, rather than steady, accumulative growth, we can expect to see unintended consequences, misalignment, ineffectiveness, and apurposiveness. Cultural phenomena like strategy will evolve through off-shoots and in the inter rather than the intra.

Deleuze and Guattari expanded on the evolution of the rhizome as a process of deterritorialization. The territory (i.e., the ensemble of relationships that constitute a rhizome) loses its context and organization only to continually change into something new through a process of reterritorialization. This process never stops and the rhizome never ceases to be; instead, it spatially expands and changes.

Since the introduction of this theoretical construct in the 1980s, there have been numerous adoptions thereof within both science in general and information systems 
in particular. For example, Choi et al. [7] utilized the non-centralized structuring of the rhizome as a model for understanding social justice movements on Twitter, Brailas [3] used it to understand technology-mediated learning, and Dalton [8] used it in the study of the emergence of assemblages of urban housing data. In Leclercq-Vandelannoitte and Aroles [22], the rhizome was used as a vehicle for understanding surveillance and the emergence of digitally enabled control societies.

We utilize the rhizome as a conceptual lens for understanding the emergence of digital transformation strategy. Seeing digital transformation strategy as an assemblage of non-linear, continuous practices of strategy (re)formulation allows us to approach the emergent characteristics of a digital transformation strategy-in-action, as depicted by Chanias et al. [6], from what we believe to be a novel, advantageous vantage point.

\section{Method}

We utilized a clinical field study approach [32] in the study of a large municipality in Sweden $(10,000$ employees, 100,000 citizens) over their past four years (2017-2021) of digital transformation. The fact that the researcher(s) were actively engaged in the organization with the goal of solving a specific problem was key to the method of clinical study $[32,49]$. The research team was approached by the municipality (a pre-digital organization as classified by Chanias et al. [6]) and asked to support them through engaged scholarship [34]. The organization had had negative experience with consultants and was under the impression that the activities that they were involved in lacked best-practice solutions. They saw a need to develop new knowledge while exploring different approaches. The idea was that the researchers would support the organization with directed studies that would inform decision-making on the fly. The overarching research program spans four years (2019-2022), and involves studies on funding, strategy, governance, digital infrastructure, and benefits realization.

So far, data has been collected in the form of steering documents (140 primarily policy-related documents in areas like governance, strategy, and organization) and interviews (60 from all levels of the organization including politicians but excluding citizens); observations (field notes), communications (emails, phone calls), and seminars have also been examined (25). We utilized a combination of purposive [11] and snowball sampling [15] to identify both the documents and interviewees in close collaboration with the organization's digital transformation team. All interviews were sound-recorded and transcribed, and they cover all departments within the organization. The study's results were continuously communicated through reports, of which there have been five so far. These reports have been instrumental for the continued digital transformation of the organization and have created ripple effects in other public sector organizations, as the reports were freely distributed to all. In terms of the clinical approach, this effect of the intervention can be equated with validation of the research [32].

The research process was iterative and involved weekly check-ins with the organization during which preliminary findings were discussed and methods of data collection were designed. Data was continuously and iteratively analyzed through primarily thematic analysis [14]; we have utilized new combinations and pieces of the growing empirical material over time as well as revisited previous analyses [33].

In terms of the analysis of the strategy-as-practice through the lens of rhizomatic theory, we used the construct of the territory as an entry point into data analysis while remaining cognizant of and attentive to aspects of the accounts that contain indications of transgressions of organizational, temporal, hierarchical, and/or personal boundaries. This involved paying particular attention to instances in which we could identify new ideas, individuals, and/or organizational functions that had not previously been mentioned. These occurrences were singled out and then analyzed chronologically, i.e., they were seen as de- and reterritorialization within the rhizome.

This project is part of the research consortium for digital government within the Swedish Center for Digital Innovation. As part of the programmatic research approach of the consortium, the project has both been fueled by and benefited from parallel clinical projects with other organizations [28].

\section{Results}

The results are presented as a chronological depiction of the emergence of a digital transformation strategy for the municipality; a chronological presentation was chosen over a conceptual one to increase readability. References to specific respondents are omitted from the results for the sake of anonymity.

\subsection{Formation (2017)}

"We started with an entirely new concept in 2017, not really knowing what we got ourselves into. "

In 2017, local politicians were experiencing a growing feeling of despair. The financial standing of the organization was increasingly dismal due to a combination of demographic changes in the population and stagnating economic growth. There was a looming 
feeling that something dramatic needed to be done to save the organization from obsoleteness. Alongside this, there was a strong push from the central government to increase the utilization of the benefits of digitalization as Sweden set the ambitious goal of becoming the best nation in the world in that regard. The combination of despair and belief in digitalization as one potential solution instigated a process whereby the politicians formulated a clear objective for digitalization as follows:

"In [omitted] municipality we utilize the benefits of digitalization to improve quality and increase safety in municipal service. Through increasing the efficiency of the municipal operations, we free up time and resources to strengthen democracy, increase participation, and autonomy for the citizens of [omitted]."

This process of formulating a politically sanctioned goal of digitalization was exemplified by a strong zeal and high level of involvement; changes in the policy were being made by involved politicians up until the last possible moment. The focus on digitalization ultimately benefiting the citizens, not just increasing the efficiency of internal operations, was central to the objective and internal efficiency was deemed to be a pre-requisite for freeing up resources.

"So, the particular goal we have is actually processed and enriched in the political process. It changed all the way to the board, where the final suggestion was presented. If I don't remember entirely wrong, the final suggestion was actually on one of those hand-written notes in a scanned version, beamed to the board. It was on that level; they had fidgeted about with it that much. And that is why it is so much more better to have put a big emphasis on values for those that we exist for. It really has been something that has been discussed quite at length in politics."

In conjunction with the new political objective of digitalization, the organization recruited a Chief Digital Officer (CDO) to lead the pending digital transformation. The organization had previously seen IT as a cost to be kept as low as possible, something governed through a strict supply-demand setup that prioritized maintaining the efficient status-quo of operations rather than transformation. The new CDO was tasked with acting on the new objective and was granted significant leeway and clout in terms of both freedom to operate and resources.

\subsection{Initiation (2018)}

"There are no incentives to increase efficiency in operations within our organizations."

The new CDO was first given responsibility over the IS department (turnover $€ 25 \mathrm{M}$ ). In parallel with this, he created a new organizational entity dubbed
Digitalization and Innovation (turnover $€ 3,5 \mathrm{M}$ ), centralizing previously decentralized innovation staff and complementing them with both new recruits and restaffing from the IS department. The new organization was tasked with becoming the speaking-partner for digitalization.

"We put great emphasis on what digitalization actually is in the conversation with operations, that it isn't anything new but actually something that all parts of operations are already engaged in. There is one difference; we will now utilize the potential of technology in business development."

The new organization noticed a lack of common ground in their dialogue with operations. They learned that they would need to propagate a uniform internal definition of digitalization given the interpretative potential of the concept. They decided to go with a definition that downplayed the societal implications and instead defined digitalization as a method for business development where digital solutions are used to either automate or innovate. In these terms, digitalization can either optimize existing operations (under the assumption that they will continue to be relevant in the future) or explore new value creation paths (under the assumption of change).

As the conversation with operations began, it became apparent that the existing thresholds for successful digitalization did not lie in technology nor competence but rather in organizational inertia. The team determined that the primary objective of the first phase of digitalization needed to be focused on increasing the transformation capability of operations.

"In order to succeed with digitalization, we need to be able to increase transformation capability, not solely in terms of competence but also in terms of the ability to work with change."

The digital transformation team initiated a round of projects that were chosen with the goal of probing operations to determine the optimal setup of the initiatives.

"From the beginning, they [operations] were a tad defensive in initiating projects. But, as we found, if you have participated in a smaller project, you feel safer, you know what it entails, you remove the worry, and then you can gradually increase the scope of the projects. What you do is train them from an operations perspective and it becomes a change journey; you actually train change."

This approach, working with small projects to acquaint operations with digital development methodology and then gradually increasing the complexity and scope of the projects, was deemed as a success. Here, the digital transformation team worked in a manner that empowered the people from operations to 
increase the transformation capability of the organization.

\subsection{Instruction (2019)}

As the work progressed, the digital transformation team developed an emergent list of core assumptions that guided them in their work. As the overarching idea was to increase the transformation capability of the organization, the team identified formalism in terms of governance and control as a direct deterrent to action. Getting an initiative up and running required a lot of paperwork and involved operations having to secure funding to internally purchase the project from the IS department. The team noticed that this practice resulted in delays for project initiation and a tendency to only focus on initiatives that had a clear return on investment and a swift payback period. This biased the selection of initiatives toward automation rather than exploration, which was deemed detrimental to transformation.

The team introduced financial slack and a process involving no internal market mechanisms to counteract formalism. Instead, the digital transformation team would work directly with operations and reduce the complexity of project initiation and management.

"We would never have been able to do this if we did not have central funds that afforded an increase in pace, ambition, and the possibility to prioritize."

To reduce complexity, the digital transformation team took it upon themselves to shield operations from the complexities of governance instead of simply disregarding it. They understood that they needed control and chose to hide the complexities of this from operations.

"Previously, we allocated the responsibility for defining and documenting the projects and solutions to operations so that they would be compliant in the procurement process. If we had continued along that path, we would have gotten nowhere."

"Just do it, let's go. There needs to be visible progress. All [initiatives] are evaluated according to the same process."

In setting up the projects, the team experimented with an approach inspired by dev-ops, i.e., crossfunctional teams of developers and operations. They found that this setup allowed them to create a closer relational proximity to operations and increase the buyin from operations in the actual adoption of a solution that was being designed through empowerment. They also found that staying close to operations and sharing the load increased their ability to identify and propagate solution designs that would be scalable to other subsets of operations.

Other core lessons learned during this period included a need for transparency in terms of what was being worked on (resulting in the digital transformation team creating a web page on which all initiatives and their statuses were presented), a need to focus on benefits realization (all projects needed to be discussed in terms of return on investment), and a need to focus on learning in cases where the team experienced failure (being open with these failures and clearly communicating lessons learned, instilling the organization with an openness to experiment).

\subsection{Expansion (2020)}

Having explored and fine-tuned a process for onboarding new parts of operations into working semiautonomously, the digital transformation team started to increase the pace of their activities by adding more resources to the digital transformation team in the form of developers expropriated from the IS department to increase the team's ability to execute projects.

"Each minute behind the desk is a minute lost. We must be out there meeting people. We should have dialogue, we should have conversations, and we should build from there."

There was a feeling among the team members that they were in a race against time. They were hard pressed not only for results but also for earning the trust bestowed upon them by an organization that was struggling financially but chose to allocate significant resources to digital transformation.

The demand for interactions with the digital transformation team was increasing exponentially, with municipal-owned companies joining in.

"The market is changing at a pace with which we are not keeping up; we could lose customers and develop problems with our finances. In some cases, we actually have to change at the same pace as society or risk falling behind and losing our competitive edge."

At the same time, the organization saw an increasing need to funnel resources toward not only development but also the modernization of digital infrastructure. The infrastructure was deemed as unable to handle the swift pace of development and it soon became apparent that infrastructure was a cost-driver and deterrent thereto. To counteract this, the team identified the need to work on the development and creation of new directions for the IS department charged with maintaining the infrastructure. Each new initiative was therefore assessed both ex-ante and ex-post in terms of implications for the digital infrastructure, and funds for modernization were transferred to the IS department. This significant change naturally led to increased collaboration between the developers and the IS department while simultaneously calling for increased pacing of development, directly counteracting the expressed need for increased pace in development. As a 
direct consequence of this, the organization decided to implement an API strategy for both its development and maintenance, resulting in (among other things) new directions for procurement and life-cycle management.

\subsection{Concentration (2021)}

"We have actually already doubled our development capacity once, and we need to double it again. That forces us to educate others, to be able to split cells, we can't be more than one person in meetings anymore or participate in all forums where there is a need in the future. Yes, but will then carry the message further?"

New challenges arose for the digital transformation team with the expansion of development and the increased emphasis on a parallel modernization of infrastructure; these were primarily related to the continued expansion of development activities to meet the continued influx of requests for dialogue and development from operations. The municipality had recently increased the budget of the organization by $30 \%$ through more investment funding. Additionally, with the CDO also controlling the funds for the IS department, the option of re-allocating development resources was always on the table. The organization utilized external consultants to fill the vacancies created in the IS department by shifting development resources from maintenance to digital transformation. Instead of, as the organization had done in the past, assigning these consultants to development, they worked within a jobrotation scheme for permanent employees. The underlying idea was to see the permanent employees as important carriers of organizational culture in a way that the external consultants were not and to ensure tight communication between digital infrastructure and development.

In scaling the digital transformation team (which was 30 full time equivalents at the time), the CDO struggled with balancing the portfolio of initiatives and the centralization/decentralization of resources.

"I still feel that we have been a tad too reserved, which we can see in the measurement of our development portfolio. We continue to need an outsidein perspective and more input from our citizens. That is no easy feat... I think that is something that municipalities struggle with, having the wrong perception of where there is resistance or what the needs of the actual citizens are. They sometimes don't even want to listen to the citizens."

Over the past three years, the organization saw an increase in externally directed initiatives (i.e., citizencentric direct value) from none to $35 \%$ of the total portfolio value in 2021. Despite this increase, the organization still struggled with prioritizing between efficiency-related value (internal, short payback period, and high return on investment) and citizen-related value (external, not efficiency-oriented, and therefore unclear in terms of return on investment).

"I guess there are two pitfalls here. One is doing something too centralized where we build a digital infrastructure that is not purposive or that does not meet demand... The other pitfall is that we develop solutions that are too isolated, things that do not form a whole and are impossible to continue to build on."

There was a clear need in centralizing in organizing for scale, but this solution also had clear trade-offs in terms of the development pace and the close relationship formed with operations. The CDO was torn between these two end-states, searching for a viable approach that would remain sustainable in the future.

\subsection{Summary of findings}

Figure 1 summarizes the status of the digital transformation strategy of the municipality as of June 2021.

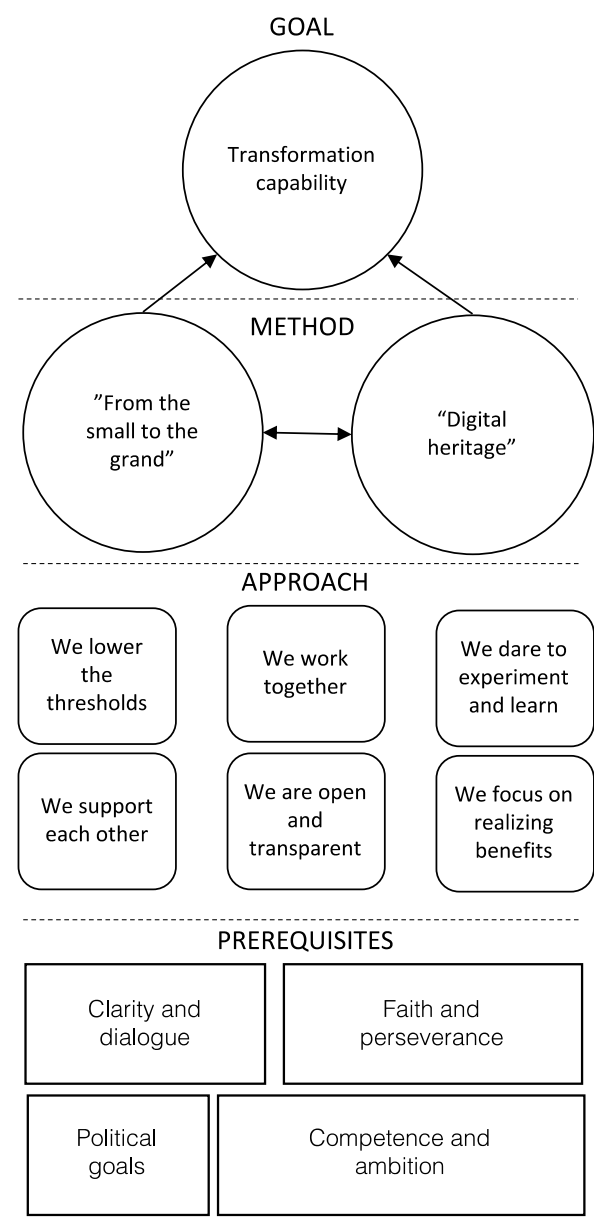

Figure 1. Illustration of current strategy (June 2021). 


\section{Discussion}

With this study addressing the research question of how a digital transformation strategy-as-practice emerges over time, we utilize the theoretical perspective of the rhizome [9] to further analyze the findings. In Figure 2, the rhizomatic strategizing is presented visually. The rhizome develops over time through offshoots that sometimes link to other off-shoots. These links form serendipitously, and sometime break over time.

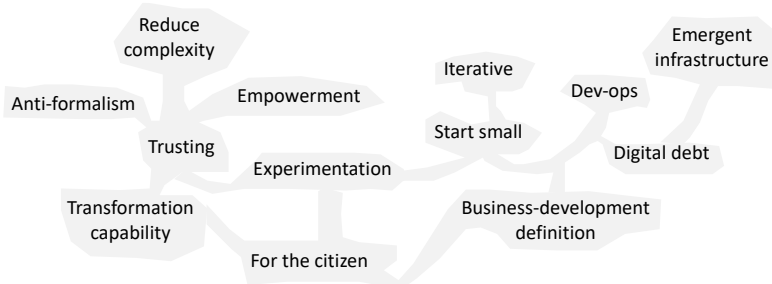

Figure 2. A map of rhizomatic strategizing in the organization.

As seen in Figure 2, the rhizomatic strategizing involved a set of constructs linked through conceptual and/or causal/temporal chains. There was no central concept for the strategizing; it instead emerged through a set of lessons that began back in 2017. There were happy coincidences, such as the digital transformation team identifying the link between citizen-centrism and experimentation as a method [36] and between experimentation and a set-up of projects in which devops [23] became key and understanding that the overarching objective should be transformation capability to counteract the inertia of the organization. The assemblage of digital transformation strategy has, in other words, amassed over time through a process of amalgamation, gradually expanding the rhizome into what it is today.

The narrative of this strategizing could be one of instrumentality and plannability, yet, as we have found it, is much more one of serendipity, similar to how Fink et al. [12] depicted strategy development in rapid innovation. This highlights links to adaptive governance, as proposed by Janssen \& Van der Voort [18], where the very setting of rhizomatic strategizing is seen as being in direct conflict with more traditional perceptions of control-oriented governance. In line with Wiener et al. [41], the organization's ability to shift between value creation- and value appropriation-based control goals and between exploitation- and exploration modes may be seen as an indication of adaptive governance being involved in the emergence of the assemblage, which constitutes rhizomatic strategizing.

Key to the assemblage, according to Deleuze and Guattari [9], is the notion of territoriality. An assemblage occupies physical/metaphysical territory, and the territory simultaneously "makes" the assemblage. In the case of the rhizomatic strategizing presented in this study, the territory transgresses the ISoperations divide, the political-service divide, and the government-citizen divide. As noted by Yoo et al. [43], digital transformation challenges previous assumptions of boundaries, instilling new boundaries and annulling old ones. Our findings show that the strategy crosses traditional boundaries by combining issues on separate sides of the divide, expanding ad infinitum into the void of society through a parallel process of de- and reterritorialization as it emerges [9].

There are two main implications of this research. First is the value of the rhizomatic approach to understanding strategy-as-practice. In this regard, we found that the non-hierarchal and anti-linear foundations of the rhizome open new avenues for thinking about the process of strategizing. The ability to break free from the linear mode of thinking and understand the latency of core ideas in what, over time, constitutes the core elements of the strategy is a powerful vehicle for analyzing developing strategies. The findings on the innate value of experimentation, facilitated by slack and both dependent upon and instilling trust between operations and the digital transformation team, are an additional contribution. Previous studies have identified the significance of the role of trust but have downplayed the generative mechanism of trust in digital transformation. Here, the mechanistic approach of digital transformation being assigned to a $\mathrm{CDO}$ and his/her team and/or the IS department was shown to be detrimental to the overarching ideas of digital transformation as proposed by Vial [35] and others.

In terms of implications in practice, we offer two main contributions. First, organizations should use this case as a source of inspiration in terms of allocating sufficient slack and trust, allowing the digital transformation strategy to emerge as opposed to simply be designed and executed. This will allow the strategizing to expand beyond previous boundaries, be they functional, conceptual, or logical, and increase the value of the final strategy. Secondly, the combination of the operations and digital transformation teams through the dev-ops approach, fueled by a reduction of (visible) complexity, should be considered a viable approach to successfully executing digital transformation.

We propose that future research continues to explore the utility of the rhizomatic perspective to strategizing through two primary projects. The first would be a longitudinal project designed to map the emergence of the strategy rhizome. We argue for studying the expansion of the rhizome through the trial-and-error approach in which it emerges. Putting equal emphasis on the failed and successful off-shoots, both their de- 
and reterritorialization, will provide a more detailed understanding of the mechanisms involved in rhizomatic strategizing. The second would be a project focused on the constraints of existing governance on rhizome formation and growth. Previous research, such as that of Magnusson et al. [24], highlighted the decrease in strategic flexibility stemming from governance and argued for strategic maneuverability and agility being a function of the absence of formal control (see also [26]); this is also supported by the research on organizational slack [29]. The project would involve mapping the role of slack in rhizomatic strategizing.

There are three main limitations to our study, all of which are related to our choice in method. First, as this is a clinical study, we actively sought to affect the process depicted here. The definition of digitalization, the ambidextrous assumptions, and the importance of slack were consequences of ongoing discussions between the research and digital transformation teams. In other words, one could argue that the study was tainted by the interference of the researchers. In the clinical approach, however, this is a positive thing [32]. Secondly, as this was a study on a single organization, we acknowledge its limitations in terms of empirical generalizability; however, we also argue, as did Eisenhardt [10], that this does not necessarily negatively impact the study's theoretical generalizability. Third, the choice to study a public sector organization, i.e., a municipality, equally implies limitations for our study. As noted by Bannister [2], studies of public sector organizations run the risk of losing out in terms of the transferability of their findings due to the institutional environment of public sectors differing substantially between nations. We argue that the clear links to particularities stemming from the Swedish governmental characteristics have little impact on the theorizing in our study nor the generalizability of our findings.

\section{Conclusion}

This study answers the research question of how a digital transformation strategy-as-practice emerges. We found that the strategy emerges in a rhizomatic order, where off-shoots de- and reterritorialize concepts, functions, and logics in a continuous process of rhizome amassment. This process is non-intentional and nonlinear, and success is serendipitous. Rhizomatic strategizing was found to be facilitated through a combination of slack resources and adaptive governance.

\section{References}

[1] K. Antonopoulou and C. Begkos, "Strategizing for Digital Innovations: Value Propositions for Transcending Market Boundaries", Technological Forecasting and Social Change, 156, 2020, pp. 120042.

[2] F. Bannister, "The Curse of the Benchmark: An Assessment of the Validity and Value of E-government Comparisons", International Review of Administrative Sciences, 73(2), 2007, pp. 171-188.

[3] A. Brailas, "Rhizomatic Learning in Action: A Virtual Exposition for Demonstrating Learning Rhizomes", Eighth International Conference on Technological Ecosystems for Enhancing Multiculturality, October 2020, pp. 309-314.

[4] Clarke, V., and V. Braun, "Thematic Analysis", Encyclopedia of Critical Psychology Springer, New York, NY, 2020, pp. 1947-1952.

[5] Cetina, K.K., T.R. Schatzki, and E. Von Savigny, eds., The Practice Turn in Contemporary Theory. Routledge. 2005

[6] S. Chanias, M.D. Myers, and T. Hess, "Digital Transformation Strategy Making in Pre-Digital Organizations: The Case of a Financial Services Provider", The Journal of Strategic Information Systems, 28(1), 2019, pp. 17-33.

[7] J.O. Choi, J. Herbsleb, J. Hammer, and J. Forlizzi, "Identity-Based Roles in Rhizomatic Social Justice Movements on Twitter", Proceedings of the International AAAI Conference on Web and Social Media, 14, May 2020, pp. 488-498.

[8] C.M. Dalton, "Rhizomatic Data Assemblages: Mapping New Possibilities for Urban Housing Data", Urban Geography, 41(8), 2020, pp. 1090-1108.

[9] G. Deleuze and D. Guattari, "A Thousand Plateaus: Capitalism and Schizophrenia”, Trans. By B. Massumi University of Minnesota, Minneapolis, 1987.

[10] K.M. Eisenhardt, "Building Theories From Case Study Research", Academy of Management Review, 14(4), 1989, pp. 532-550.

[11] I. Etikan, S.A. Musa, and R.S. Alkassim, "Comparison of Convenience Sampling and Purposive Sampling", American Journal of Theoretical and Applied Statistics, 5(1), 2016, pp. $1-4$.

[12] T.M.A Fink, M. Reeves, R. Palma, and R.S. Farr, "Serendipity and Strategy in Rapid Innovation", Nature Communications, 8(1), 2017 pp. 1-9.

[13] M. Fitzgerald, N. Kruschwitz, D. Bonnet, and M. Welch, "Embracing Digital Technology: A New Strategic Imperative," MIT Sloan Management Review, 55(2), 2014, pp. 1 .

[14] P. Florina and A. Mitan, "Social Media and Marketing of the "Popcorn" Music Wave: The Success of Romanian Commercial Musicians Analysed Through Their Perceived Image on Facebook and Youtube", Economics \& Sociology 5, no. 2A, 2012, pp. 125.

[15] L.A. Goodman, "Snowball Sampling", The Annals of Mathematical Statistics, 1961, pp. 148-170.

[16] P. Gottschalk, 1999, "Implementation of Formal Plans: The Case of Information Technology Strategy", Long Range Planning, 32(3), 1999, pp. 362-372.

[17] D.C. Hambrickand P.A. Mason, "Upper Echelons: The Organization as a Reflection of its Top Managers", Academy of Management Review, 9(2), 1984, pp. 193-206. 
[18] M. Janssen and H. van der Voort, "Adaptive Governance: Towards a Stable, Accountable, and Responsive Government", Government Information Quarterly, 1(33), 2016, pp. 1-5.

[19] P. Jarzabkowski, J. Balogun, and D. Seidl, "Strategizing: The Challenges of a Practice Perspective", Human Relations, 60(1), 2007, pp. 5-27.

[20] Jarzabkowski, P., Strategy as Practice: An Activity-Based Approach. Sage. 2005.

[21] J.K. Lê and P.A. Jarzabkowski, "The Role of Task and Process Conflict in Strategizing", British Journal of Management, 26(3), 2015, pp. 439-462.

[22] A. Leclercq-Vandelannoitte and J. Aroles, "Does the End Justify the Means? Information Systems and Control Society in the Age of Pandemics", European Journal of Information Systems, 29(6), 2020, pp. 746-761.

[23] Lwakatare, L.E., P. Kuvaja, and M. Oivo, 2015, May. Dimensions of devops. In International conference on agile software development (pp. 212-217). Springer, Cham.

[24] J. Magnusson, D. Koutsikouri, and T. Päivärinta, "Efficiency Creep and Shadow Innovation: Enacting Ambidextrous IT Governance in the Public Sector", European Journal of Information Systems, 29(4), 2020, pp. 329-349.

[25] I. Mergel, N. Edelmann, and N. Haug, "Defining Digital Transformation: Results from Expert

Interviews", Government Information Quarterly, 36(4), 2019, pp. 101385.

[26] B. Müller-Stewens, S.K. Widener, K. Möller, and J.C. Steinmann, "The Role of Diagnostic and Interactive Control Uses in Innovation", Accounting, Organizations, and Society, 80, 2020, pp. 101078.

[27] P. Parviainen, M. Tihinen, J. Kääriäinen, and S. Teppola, "Tackling the Digitalization Challenge: How to Benefit From Digitalization in Practice", International Journal of Information Systems and Project Management, 5(1), 2017, pp. 63-77.

[28] S. Ram and P. Goes, "Focusing on Programmatic High Impact Information Systems Research, not Theory, to Address Grand Challenges", MIS QUARTERLY, 45(1), 2021, pp. 479-483.

[29] Y. Rahrovani, A. Pinsonneault, and R.D. Austin, "If You Cut Employees Some Slack, Will They Innovate?" MIT Sloan Management Review, 59(4), 2018, pp. 47-51.

[30] J. Reis, M. Amorim, N. Melão, and P. Matos, "Digital Transformation: A Literature Review and Guidelines for Future Research", World Conference on Information Systems and Technologies, March 2018, pp. 411-421. Springer, Cham. [31] A. Lanamäki, K. Väyrynen, S. Laari-Salmela, and M. Kinnula, "Examining Relational Digital Transformation Through the Unfolding of Local Practices of the Finnish Taxi Industry", The Journal of Strategic Information Systems, 29(3), 2020, pp. 101622.

[32] Schein, E.H., The Clinical Perspective in Fieldwork, Sage Publications, Inc., 1987.

[33] Silverman, D., Interpreting Qualitative Data, Sage, 2015. [34] Van de Ven, A.H., Engaged Scholarship: A Guide for Organizational and Social Research, Oxford University Press on Demand, 2007.
[35] G. Vial, "Understanding Digital Transformation: A Review and A Research Agenda", The Journal of Strategic Information Systems, 28(2), 2019, pp. 118-144.

[36] L. Wang, S. Bretschneider, and J. Gant, "Evaluating Web-Based E-government Services With a Citizen-Centric Approach", Proceedings of the 38th Annual Hawaii International Conference on System Sciences, January 2005, pp. 129b-129b. Ieee.

[37] K.S. Warner, and M. Wäger, "Building Dynamic Capabilities for Digital Transformation: An Ongoing Process of Strategic Renewal", Long Range Planning, 52(3), 2019, pp. 326-349.

[38] R. Whittington, "Environmental Structure and Theories of Strategic Choice", Journal of Management Studies, 25(6), 1988, pp. 521-536.

[39] R. Whittington, "The Work of Strategizing and Organizing: For a Practice Perspective", Strategic Organization, 1(1), 2003, pp. 117-125.

[40] R. Whittington, B. Yakis-Douglas, K. Ahn, and L. Cailluet, "Strategic Planners in More Turbulent Times: The Changing Job Characteristics of Strategy Professionals, 19602003”, Long Range Planning, 50(1), 2017 pp. 108-119.

[41] M. Wiener, M. Mähring, U. Remus, C. Saunders, and W.A. Cram, "Moving is Project Control Research Into the Digital Era: The "Why" of Control and the Concept of Control Purpose", Information Systems Research, 30(4), 2019, pp. 1387-1401

[42] M.F. Wiersema and K.A. Bantel, "Top Management Team Demography and Corporate Strategic Change", Academy of Management Journal, 35(1), 1992, pp. 91-121.

[43] Y. Yoo, O. Henfridsson, and K. Lyytinen, "Research Commentary - the New Organizing Logic of Digital Innovation: An Agenda for Information Systems Research", Information Systems Research, 21(4), 2010, pp. 724-735.

[44] T. Hess, C. Matt, A. Benlian, and F. Wiesböck, "Options for Formulating a Digital Transformation Strategy", MIS Quarterly Executive, 15(2), 2016.

[45] A. Correani, A. De Massis, F. Frattini, A.M. Petruzzelli, and A. Natalicchio, "Implementing a Digital Strategy:

Learning From the Experience of Three Digital

Transformation Projects", California Management

Review, 62(4), 2020, pp. 37-56.

[46] A. Danuso, F. Giones, and E.R. da Silva, "The Digital Transformation of Industrial Players: A Guide", Business Horizons, 2021

[47] Arvidsson, V. and J. Holmström, Digitalization as a Strategy Practice: What is There to Learn From Strategy as Practice Research? The Routledge Companion to Management Information Systems, Routledge, pp. 218-231, 2017.

[48] A. Bharadwaj, O.A. El Sawy, P.A. Pavlou, and N. Venkatraman, "Digital Business Strategy: Toward a Next Generation of Insights", MIS Quarterly, 37(2), 2013, pp. 471482.

[49] R. Baskerville, J. vom Brocke, L. Mathiassen, and H. Scheepers, "Special Issue Call for Papers: Clinical Research from Information Systems Practice", European Journal of Information Systems, 2020. 